\title{
IL FANTASTICO NELLA DRAMMATURGIA ITALIANA DEL PRIMO NOVECENTO
}

\author{
Nicola Pasqualicchio \\ nicola.pasqualicchio@univr.it \\ Università degli Studi di Verona
}

Recibido: 02-07-2014

Aceptado: 19-11-2014

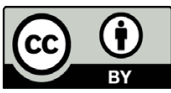

SOMMARIO

L'articolo propone un panorama sintetico della presenza del fantastico nella drammaturgia italiana del primo Novecento, in particolare negli anni Dieci e Venti, con il proposito di dimostrare che non è solo attraverso la narrativa, ma anche appunto attraverso la scrittura per il teatro che l'Italia si ricava nel xx secolo quel posto originale nell'ambito del fantastico che non aveva invece raggiunto nel secolo precedente. L'analisi prende le mosse da un'opera di Enrico Annibale Butti che unisce influssi gotici e di Poe con una sensibilità simbolista e una propensione per l'occultismo, per passare poi in rassegna il contributo offerto al fantastico dal teatro sintetico futurista. In seguito lo studio si sofferma sul cosiddetto «teatro del grottesco», prendendo in particolare considerazione l'opera di Luigi Antonelli, per concludersi con l'esame dell'applicazione al teatro del «realismo magico» teorizzato da Massimo Bontempelli.

Parole Chiave: teatro fantastico, drammaturgia italiana del primo Novecento, teatro futurista, teatro del grottesco, realismo magico

\section{Abstract}

The article offers a concise outline of the presence of fantastic in Italian early twentieth-century drama, with the intention to demonstrate that it was not only through narrative, but also through dramatic composition that Italian fantasy made a place for itself in the last century. The analysis focuses in particular on four moments in this theatrical trend: a play of Enrico Annibale Butti strongly influenced by Poe; the occult side of futurist «teatro sintetico» (synthetic theatre); the group of «grotesque» playwri- 
ghts, with a particular attention to Luigi Antonelli's work; and the presence of «magical realism» in Massimo Bontempelli's plays.

KEYwORDs: fantastic theatre, early twentieth-century Italian drama, futurist theatre, theatre of «grotesque», magic realism.

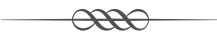

Solo nel Novecento la letteratura italiana approda in maniera pienamente originale al modo fantastico, quasi totalmente estromesso, alla stregua di un corpo estraneo, dalla cultura peninsulare nella prima metà dell'Ottocento, e nei decenni immediatamente successivi recuperato più che altro come tardivo echeggiamento di modelli stranieri, da Poe ai romantici tedeschi e francesi, nell'ambito della scapigliatura e di quelle frange di letteratura verista affascinata, con l'alibi dell'atteggiamento scientista, da esperienze medianiche e fenomeni paranormali. È dunque all'inizio del secolo scorso che si apre una via peculiarmente italiana al fantastico moderno, in un clima culturale complesso, in cui propaggini simboliste, ribellioni avanguardiste, ritorni all'ordine talora si oppongono, talaltra interagiscono e si sovrappongono. Pur in modo variegato e non senza eccezioni, il fantastico italiano sembra allora principalmente indirizzarsi alla manifestazione di una sostanziale ambiguità del reale, di una sospensione tra la vita materiale e il sogno, e all'accoglienza di istantanee epifanie che d'improvviso rovesciano il senso delle cose quotidianamente esperite, rivelandone la coappartenenza a una dimensione non apertamente soprannaturale né smaccatamente demoniaca, ma più sottilmente e magicamente inquietante. Che tra i rappresentanti riconosciuti del fantastico italiano d'inizio Novecento figurino (e anzi primeggino) Massimo Bontempelli e Luigi Pirandello è poi, ai fini dell'argomento del presente saggio, un dato importante, essendo essi drammaturghi non meno che narratori, e attraversando la loro corda fantastica la produzione per il teatro non meno di quella destinata alla sola lettura. E non si tratta di un caso: ciò che qui vorremmo evidenziare - più di quanto non si sia fatto finora - è infatti che, se l'Italia del Novecento si ricava una propria originale posizione nell'evoluzione del modo fantastico, lo deve anche al teatro. A questo scopo passeremo brevemente in rassegna quelle che ci sembrano alcune tappe esemplari, tra gli anni Dieci e i Trenta del Novecento, di tale reciproca implicazione di teatro e 
fantastico sulle scene italiane: Enrico Annibale Butti, i futuristi, il gruppo dei «grotteschi», Bontempelli. La complessità e la grandezza della figura di Pirandello, anche solo in relazione al suo rapporto con il fantastico, di cui sempre più chiaramente si riconosce la presenza e l'importanza tanto nella narrativa che nel teatro, comporterebbero su di lui una riflessione a parte: per questo il presente contributo si ferma alle soglie della sua opera, in cui sono sussunti e portati a un più alto livello di consapevolezza teorica e compiutezza artistica molti dei motivi che ci accingiamo ad analizzare nei suoi predecessori e contemporanei.

1.

Prendiamo le mosse da un'opera che risalta più come occasione di tardivo recupero di una concezione gotico-romantica del fantastico, pur se attraversata da inquietudini simboliste e da moderne consapevolezze psicologiche, che come momento rappresentativo dei nuovi umori novecenteschi: Il castello del sogno, poema tragico di Enrico Annibale Butti, pubblicato nel 1910 e rappresentato postumo nel 1914. Il drammaturgo milanese, vissuto tra il 1868 e il 1912, oggi dimenticato ma assai noto e rappresentato a cavallo tra i due secoli, fu in prevalenza autore di opere improntate a uno psicologismo di derivazione ibseniana (De Antonellis, 2008); ma è proprio con l'atipico dramma fantastico in endecasillabi sciolti che ne concluse la carriera di drammaturgo, che egli confidò di aver creato il proprio capolavoro. Tale certamente non appare agli occhi di un lettore moderno, e c'è da dire che neppure la critica contemporanea lo accolse con entusiasmo: eppure il testo, sia per gli influssi svariati che mette originalmente in gioco che per le soluzioni drammaturgiche adottate in relazione al fantastico, costituisce un oggetto di studio di notevole interesse. Tra le fonti ispiratrici, quella che si palesa immediatamente è un racconto tra i più celebri e più belli di Edgar Allan Poe, La caduta della casa Usher (The Fall of the House of Usher, 1839). Il debito verso lo scrittore americano fu notato già al tempo della pubblicazione del dramma (tanto che Butti dovette difendersi da accuse di plagio nei confronti del racconto) e poi ribadito da alcuni recensori della rappresentazione teatrale (Melani, 2006: 135-136). Al livello generale della trama la derivazione è in effetti evidente, ma altrettanto nette sono le differenze, che indirizzano le due opere verso esiti di senso profondamente diversi. Come Roderick Usher, il Fantasio di Butti vive isolato in un castello, assieme alla sorella Ebe alla quale è legato da un affetto morboso. Al castello giunge in visita, così come accade in Poe, un amico di vecchia data di Fantasio, Angelo. Ebe, al pari della Madeline del racconto 
americano, è gravemente malata; durante la permanenza dell'ospite la donna cadrà vittima di una crisi che la farà credere morta e ne causerà il prematuro seppellimento nei sotterranei del castello. A compimento di una sorta di delirante profezia del fratello, che ne prevede la «resurrezione», Ebe riemergerà dalle viscere della terra nelle sembianze di uno spettro coperto di sangue in conseguenza della lotta sovrumana contro la porta metallica che la chiudeva nella cripta mortuaria. Se ci limitiamo alla descrizione di questi elementi, l'accusa di plagio da parte di Butti nei confronti di Poe appare ineccepibile. Ma essi, come si diceva, si correlano con una serie di altri elementi che risemantizzano i debiti verso Poe in tutt'altra direzione di senso. Molto diversi, innanzitutto, sono i caratteri e le funzioni dei tre protagonisti. Fantasio non è affatto una replica dell'artista fragile, ultrasensibile, costantemente attraversato da turbamenti e presentimenti da visionario, incarnato da Roderick; è, per citare il poeta che egli ha sostituito alla Bibbia, un superbo misantropo che tiene l'umanità «a gran dispitto», ${ }^{1}$ un aristocratico che si isola in un autosufficiente reame di sogno in odio del «triste Mondo, dove si lavora, / si suda, si battaglia inutilmente / per odio insano o per fallace amore, / indi si muore!»; mondo in cui le relazioni umane sono «ignobili contatti», portatori «di miserie, / di lutti e di vergogne!» (Butti, 1910: 46; vv. 554-560). Anch'egli, similmente al protagonista del racconto, intrattiene un contatto con una dimensione arcana e invisibile; ma, mentre per Roderick questa caratteristica è quasi una congenita condanna caratteriale, che lo espone passivamente alle superstizioni suggeritegli dalla sua antica dimora, Fantasio è un mago, che pratica scientemente i rituali di contatto con l'aldilà e con il mondo dei morti (ed è in questo senso un precursore di quei solitari stregoni «dimissionari» dal mondo che popolano il teatro di Luigi Antonelli e culminano nel Cotrone dei pirandelliani Gi-

1 Dante, Inferno, X, 36.

2 Nel secondo canto (vv. 1106-1364) si assiste, nel momento a cui Butti destinava evidentemente il maggior impatto spettacolare della sua pièce, a una vera e propria scena di necromanzia, denominata dall'autore «orgia fantastica»: evocati da Fantasio, i morti di un cimitero escono dalle loro tombe alla presenza dell'incredulo e terrorizzato Angelo. I connotati visivi della scena («Vedi fumar la terra ed annebbiarsi / l'aria, come se lunghi esili veli / fluttuassero al vento della notte / di tra i cippi e le croci? Or vaghe forme / inconcrete si librano nel fioco / chiarore... Osserva! Osserva! Un globo bianco!... / Una gran bolla iridescente!... Un'ombra!... / Una figura non compiuta ancora: / la testa, il collo, un drappo che dilegua / nello spazio!... Gli spettri! Ecco gli spettri!» (Butti, 1910: 103-104; vv. 1283-1292) suggeriscono riferimenti tanto agli spettacoli popolari di fantasmagoria quanto all'«iconografia» delle sedute spiritiche, molto frequentate all'epoca da artisti e intellettuali (per un certo periodo Butti ne organizzò regolarmente nella propria casa (Melani, 2006: 134). Una descrizione lasciataci dallo scrittore Luigi Capuana di una seduta medianica alla quale assistette assieme a Pirandello riferisce di fenomeni analoghi a quelli suscitati dalla magia di Fantasio: «globi fosforescenti che erravano sotto la volta dello stanzone», «il profilo di un fantasma su l'alto della tenda», «la tenda [...] spinta fin sulle nostre teste quasi gonfiata da un forte vento» (Cigliana, 2006: 202). 
ganti della montagna). I suoi eccessi di attenzione percettiva, acustica soprattutto, mimano quelli di Roderick, ma solo nella scena del ritorno alla vita di Ebe hanno la funzione di paranormali auscultazioni, derivando per il resto da un'inesausta volontà di protezione da disturbi o violazioni della sua solitudine. Per quanto riguarda il rapporto con la sorella, mentre il morboso attaccamento di Roderick a Madeline suggerisce una parità gemellare tra i due che ce li fa apparire come le separate incarnazioni di un'unica anima malata, Fantasio sostituisce all'atteggiamento fraterno quello di un padre-padrone protettivo al punto di trasformarsi in un carceriere incestuosamente possessivo e geloso.

Madeline, d'altronde, è un personaggio-apparizione, altrettanto fantasmatica in vita che dopo il ritorno dalla (presunta) morte: prima della sua sepoltura e della raccapricciante ricomparsa finale, attraversa la scena del racconto una sola volta, quasi scivolando lungo una parete di fondo, muta, inconsapevole di ciò che l'attornia, ignara della presenza dell'ospite. Rispetto a questa straordinaria presenza-assenza, Ebe recupera i tratti convenzionali del personaggio femminile che la clausura impostale dal fratello rischia di uccidere: la sua malattia altro non è che sottrazione alle gioie della vita, della giovinezza, dell'amore, che le si manifesteranno tutte in un colpo grazie all'inatteso quanto terapeutico arrivo dell'ospite il quale, da buon principe azzurro, suggellerà infine la propria azione salvifica portando via con sé l'innamorata in sella al suo cavallo.

Angelo, come l'io narrante di Poe, è un vecchio amico del castellano; $\mathrm{ma}$, mentre l'ospite del racconto è invitato e desiderato, il personaggio di Butti s'intromette come un inaspettato corpo estraneo nella dorata prigione in cui si è relegato Fantasio; il quale ha ben ragione di temerlo, perché l'intruso, per quanto animato da buone intenzioni, non può che porsi in antitesi, in quanto rappresentante della vita vissuta e del mondo reale, all'artificioso e lugubre castello di chimere nel quale Fantasio ha rinchiuso Ebe e sé stesso. Il ruolo di Angelo non si limita dunque a quello di puro testimone che riveste l'io narrante della Casa Usher; risultando al contrario decisivo a livello diegetico proprio nell'imprimere agli avvenimenti quella piega che porterà al crollo (metaforico, in questo caso) del castello dei sogni.

È in particolare nella scena decisiva in cui Fantasio avverte telepaticamente il risveglio di Ebe dal sonno catalettico e la sua spaventosa lotta per sottrarsi alla prigionia della tomba, concludentesi con l'effettiva comparsa della sorella sconvolta e coperta di sangue, che il debito nei confronti di Poe si lascia più apertamente riconoscere, approdando alla citazione letterale nel 
momento di acme della tensione, un istante prima dell'apparizione della sorella: l'esclamazione di Fantasio («Insensato! / Io ti dico che in questo istante mia / sorella è dietro quella porta!» (Butti, 1910: 215; vv. 2603-2605) è infatti puntuale restituzione della fonte: «Madman! I tell you that she now stands without the door!» (Poe, 1982: 245). Anche qui, tuttavia, Butti non si adagia passivamente sul testo del racconto, ma lo varia quel tanto che serve per conferirgli una specifica e più immediata efficacia sul piano scenico. Va interpretata in questa prospettiva la sostituzione dello stratagemma di Poe di far corrispondere i suoni che provengono dai sotterranei a quelli che compaiono nel racconto fiabesco che l'amico sta leggendo ad alta voce, con la narrazione - di più sicuro impatto teatrale in quanto interamente affidata all'empito visionario di Fantasio - delle varie tappe della «resurrezione» di Ebe, punteggiata dalle esclamazioni prima di incredulità, poi di crescente sgomento di Angelo, e dalle didascalie concernenti i rumori provenienti da fuori scena, sempre più sinistramente in sintonia con quanto riferito dall'apparente delirio di Fantasio. Particolarmente efficace in questo frangente è l'idea di Butti, non derivata da Poe, di affidare per intero al tempo verbale futuro il resoconto che Fantasio fa della propria visione, conferendole perciò in modo ingannevole il carattere di una profezia, mentre gli eventi descritti si stanno volgendo al presente fuori dalla vista dei personaggi e degli spettatori, in uno spazio esterno la cui distanza rispetto allo spazio scenico si fa però sempre più ridotta, fino ad annullarsi con la comparsa in scena della rediviva Ebe. Cadono contemporaneamente, al culmine della tensione drammatica, due protettivi «allontanamenti»: la reclusione spaziale del fenomeno spaventoso fuori dalla scena, e il suo differimento temporale in un momento a venire: «in questo istante mia sorella è dietro quella porta».

In questa scena clou del dramma la pressione progressiva sullo spazio visibile di un'inquietante apparizione collegata alla Morte deriva certo direttamente da Poe, ma nel momento in cui si fa teatro suggerisce debiti o quanto meno affinità (pur nell'assenza delle morbidezze stilistiche e della rarefatta atmosfera del drammaturgo belga) con L'intrusa (L'Intruse, 1890) di Maeterlinck. È a questo proposito da rilevare che solo una parte degli elementi fantastici di cui l'opera è diffusamente intessuta derivano dalla Caduta della casa Usher: ne è di sicuro indipendente l'«orgia fantastica» del secondo canto (vedi nota 2), la cui concezione sembra rifarsi più al melodramma gotico che alle atmosfere dello scrittore americano; mentre si rendono evidenti qua e là echi faustiani («Dimmi: vuoi / tu che l'Attimo sia l'Eternità / e il Punto l'Infinito? Vuoi vedere / oltre agli astri, al di là dei monti, in fondo / ai mari, dentro le 
anime silenti?» (Butti, 1910: 94; vv. 1138-1142), il già menzionato riverbero dell'Intrusa di Maeterlinck, ma anche quello di Pelléas et Mélisande, riutilizzi di situazioni dantesche (la Dama Velata chiamata fuori dal gruppo silente delle anime e personalmente interpellata (Butti, 1910: 106; vv. 1317-1330), nonché il riferimento ad almeno un altro racconto di Poe, La mascherata della morte rossa (The Masque of the Red Death, 1842), il cui protagonista Prospero condivide con Fantasio l'arrogante sicurezza di tener fuori dal proprio castello la realtà della vita quanto la minaccia della morte, «ospite non chiamata e non attesa» (Butti, 1910: 191; v. 2255).

2.

Nonostante gli esiti del tutto divergenti della loro opera, Butti e Filippo Tommaso Marinetti, che furono legati da una lunga frequentazione amichevole e da reciproca stima, condivisero tra l'altro due spiccati interessi che contribuirono spontaneamente a indirizzare una parte della loro opera verso il fantastico: quello per il simbolismo (e per il teatro di Maeterlinck in particolare e quello per l'occultismo. ${ }^{3}$ Le radici simboliste dell'avanguardia futurista, più solide di quanto comunemente si pensi, predispongono in effetti Marinetti e tutto il suo movimento ad aperture verso una dimensione sovrasensibile arcanamente rivelantesi negli interstizi della realtà materiale che, se da una parte giustificano gli interessi per le teorie esoteriche e per i fenomeni medianici da parte di non pochi futuristi, imprimono anche tracce evidenti in parte della produzione artistica, e segnatamente teatrale, del movimento. Sia pur indicandola come un'area decisamente minoritaria, Mario Verdone rilevava all'interno del «teatro sintetico» l'esistenza di un gruppo di testi definibili come occultisti e magici, che riconosceva come quelli più legati ai precedenti simbolisti (Verdone, 1969: 92-96), e quindi, «per struttura e motivazione» (Verdone, 1969: 94), leggermente attardati rispetto alle sintesi drammatiche più decisamente avanguardiste.

Ve n'è uno addirittura, Il pesce d'aprile di Paolo Buzzi ${ }^{4}$ (Marinetti, Settimelli e Corra, 1915: 69-72), che si presenta come versione teatrale di un virtuale racconto della tradizione fantastica ottocentesca. Il minidramma, canonicamente diviso in tre brevissimi atti, inizia con la richiesta fatta a un

3 «Riprendere le esperienze spiritiste nei saloni a tendaggi meditanti di E. A. Butti quella notte stessa rileggendo le opere di Maeterlinck e al pianoforte Pelléas et Mélisande» (Marinetti, 1969: 48). Sulle propensioni occultistiche ed esoteriche di Marinetti, e più in generale del futurismo, si vedano Cigliana, 2006, e Bertolotti, 2013.

4 Questa sintesi, come le tre di Marinetti di cui si tratterà tra breve, apparve per la prima volta nel primo volume del Teatro futurista sintetico, curato da Marinetti, Settimelli e Corra e pubblicato nel 1915. 
monsignore da parte di una sconosciuta dama in nero di recarsi al capezzale di un grande peccatore in punto di morte per recargli i conforti spirituali. Il prete non ha motivi per sospettare qualcosa di strano; salvo credere a uno scherzo (è il primo d'aprile) quando, recatosi nel palazzo che la donna gli ha indicato, ne trova il nobile proprietario in perfetta salute, anche se effettivamente desideroso di scaricare dai peccati la propria coscienza. Il ministro di Dio, dovendosi occupare di un caso più urgente, rimanda la confessione alla sera; ma nel frattempo il marchese sarà morto di un colpo, e il sacerdote, ammesso nella camera dove giace il cadavere, riconoscerà la dama in nero in un grande ritratto che vi sta appeso: si tratta, gli spiega il cameriere, della moglie del signore, morta venticinque anni prima. Tutto è condotto, nella breve pièce, secondo i canoni del fantastico moderno nel senso stabilito dalla critica del secondo Novecento: l'ambientazione realistica, l'atteggiamento razionalista del protagonista, il susseguirsi di alcuni eventi strani ma di per sé non impossibili, la rivelazione finale che orienta tutto verso l'interpretazione soprannaturale. L'atteggiamento «futuristicamente» operativo ed efficace è qui in realtà concentrato proprio nella sinteticità del dramma: se è vero, come spesso è stato notato, che al tipo di fantastico di cui stiamo parlando giova straordinariamente l'adozione della forma narrativa breve, la misura ridottissima del teatro sintetico, escludendo verbosità e digressioni, si propone come la più adatta a trasferire sul piano scenico gli effetti estetici del racconto fantastico.

Altre sintesi tendono invece a una manifestazione evocativa (e anche per questo affidata a risorse non solo drammaturgiche, ma anche o prevalentemente sceniche) piuttosto che narrativa del lato in ombra della realtà. È quanto accade talvolta nei cosiddetti «drammi di oggetti», che presuppongono animisticamente la possibilità di una vita degli oggetti autonoma, e potenzialmente minacciosa, nei confronti della dimensione umana. Prevale talvolta, in quest'ambito, un atteggiamento ludico, tendente più al paradosso e all'assurdo che all'inclinazione perturbante del fantastico. Come in Pirandello, ${ }^{5}$ anche nel futurismo il comico (o l'umorismo) e il fantastico si presentano entrambi come processi di smascheramento delle convenzioni accettate (sia sul piano gnoseologico che su quello sociale), che condividono tanto i presupposti quanto gli obiettivi, ${ }^{6}$ ma pervengono a modalità diverse di «straniamen-

5 «Non c'è scelta: per guardare dietro le maschere bisogna imparare a far ridere o a incutere terrore. In quanto alleato naturale dell'umorismo, il fantastico si colloca dunque al centro del progetto letterario di Pirandello» (Pedullà, 2010: XVII).

6 «[...] identico rimane il piano di attacco alle sicurezze del positivismo ottocentesco: 1'insistenza sulle 
to» nei confronti del reale; e ancora come in Pirandello ${ }^{7}$ il modo comico risulta quantitativamente prevalente su quello fantastico. Questo non smorza i particolari effetti di perturbamento derivanti dal fantastico, quando è su di esso che si indirizza la scelta dell'autore futurista:

[...] se con l'iniezione di elementi comici l'apparizione dello strano, del mostruoso, del deforme, dell'inusitato, di tutto ciò che contrasta con le leggi naturali, viene in qualche modo privata delle sue valenze sacrali, precipitando nel bizzarro e nell'assurdo, altrove invece il contatto col soprannaturale, perdute le sue connotazioni ludiche, si riaffaccia come rottura dell'ordine costituito, come polarità inammissibile nella realtà quotidiana, riattivando nello spettatore l'inquietudine nei confronti dell'inesplicabile. (Artioli, 1975: 198)

Così, tra i drammi di oggetti composti da Marinetti si potrebbe collocare sul versante umoristico, almeno in senso lato e non senza un'ombra inquietante, un'opera come Il teatrino dell'amore (Marinetti, Settimelli e Corra, 1915: 24-27), dove al dialogo degli umani, coinvolti in una convenzionale situazione adulterina, fanno da contraltare le battute - punteggiate da scricchiolii «recitati» - del buffet e della credenza, che registrano con impassibile precisione didascalica dei dati di fatto superflui o evidenti («Cric cric. La pressione del servizio d’argenteria è superiore alla mia coesione! [...] Crac. La schiena del padrone aderisce a poco a poco alla spalliera della poltrona. [...] Cric. Piove [...] Craac. Mi dilato» (Marinetti, Settimelli e Corra, 1915: 24-26). Decisamente fantastica, invece, è l'ispirazione di Vengono (Marinetti, Settimelli e Corra, 1915: 29-31), altro dramma di oggetti di Marinetti, le cui uniche battute consistono in una serie di ordini e contrordini dati da un maggiordomo a un gruppo di servi che devono apparecchiare una tavola per un pranzo: alla fine i commensali attesi non giungono, ma in compenso le otto sedie e una poltrona, più volte spostate durante l'azione scenica, decidono di andarsene:

Un riflettore invisibile proietta sul pavimento le ombre della poltrona e delle sedie. Ombre spiccatissime, che (spostandosi lentamente il riflettore) vanno visibilmente allungandosi verso la porta-finestra.

I servi, accoccolati in un angolo, aspettano tremanti, con angoscia evidente, che le sedie escano dalla sala. (Marinetti, Settimelli e Corra, 1915: 31).

contraddizioni, il recupero di una realtà profonda occultata sotto l'ordinato scorrere dei fenomeni ai quali la scienza vorrebbe ridurre la vita, il balenare di una condizione "altra" che non sarebbe possibile nemmeno intuire con gli strumenti dell'arte piattamente mimetica» (Pedullà, 2010: XVI).

7 «Umorismo e fantastico [...] a volte possono entrare in conflitto come due Muse gelose, che pretendono dallo scrittore una fedeltà esclusiva. [...] E quando questo succede [...] è quasi sempre il fantastico ad avere la peggio» (Pedullà, 2010: XVIII). 
Marinetti ha creato questa sintesi a partire dall'osservazione degli «atteggiamenti impressionanti e pieni di misteriose suggestioni che i mobili in genere, e in particolar modo le sedie e le poltrone, assumono in una stanza dove non sono esseri umani» (Marinetti, Settimelli e Corra, 1915: 31). Mentre nel Teatrino dell'amore la vita dei mobili scorreva parallela a quella degli uomini e da essi inavvertita, qui l'animazione degli oggetti è percepita dai servi, che ne sono terrorizzati. Ma il commento finale apposto dallo stesso Marinetti in calce al dramma, spiega anche l'intenzione «registica» dell'autore che «le otto sedie e la grande poltrona, nei diversi mutamenti delle loro posizioni successivamente preparate per ricevere gli attesi» acquistino «a poco a poco una strana vita fantastica» (Marinetti, Settimelli e Corra, 1915: 31). Il senso perturbante di vita dell'oggetto inanimato non dev'essere perciò offerto agli spettatori tutto d'un colpo nel finale «scivolamento» delle ombre, ma deve già velatamente presentarsi e accrescersi nel corso dell'azione. Anche se l'autore non si sofferma a spiegarlo, questo obiettivo doveva probabilmente essere raggiunto attraverso il rapporto tra collocazione spaziale e particolare illuminazione degli oggetti e forse corroborato da un'evoluzione del rapporto fisico tra i servi e le sedie: ricorrendo comunque a risorse prettamente sceniche. Proprio questa indeterminatezza nell'indicare le modalità allestitive di quanto è più essenziale all'efficacia fantastica della sintesi, dipendente anche dalla scarsa confidenza di Marinetti (e di gran parte dei drammaturghi futuristi) con la pratica scenica, fa al postutto di Vengono l'espressione di una pura intenzionalità fantastica, di cui si avverte come un difetto la vaghezza della soluzione realizzativa.

Più che nei drammi di oggetti, è forse in alcune sintesi basate sull'intersecazione di situazioni tra loro estranee, e magari appartenenti a diversi livelli di realtà, che il teatro futurista raggiunge il maggior grado di suggestione fantastica. Ne scegliamo un esempio ancora di Marinetti, Un chiaro di luna (Marinetti, Settimelli e Corra, 1915: 32-33), che l'autore definisce "compenetrazione alogica». La situazione è semplicissima: due innamorati su una panchina, in una notte di luna, si scambiano effusioni amorose. D'improvviso un signore "grasso e panciuto», invisibile ai due, si siede accanto a loro: li guarda, controlla il proprio orologio, pronuncia degli «hum» forse di perplessità, si alza e passeggia per un po', si risiede sulla panchina. Infine si rialza, passa dietro la panchina, tocca lievemente la spalla prima di lei poi di lui e si allontana lentamente. In corrispondenza della sua apparizione i due giovani avvertono qualcosa di strano: la sensazione di un alito di vento nell'aria ferma, il dubbio che il giardino non sia più deserto, un brivido di freddo che coglie 
la ragazza. Con assoluta discrezione e al di fuori di qualsiasi stereotipo spettrale, Marinetti riesce qui a creare un'atmosfera sottilmente perturbante, incarnando in questo fantasma panciuto e bofonchiante (che non è affatto comi$\mathrm{co}$, anche se basterebbe un nulla per renderlo tale) una minaccia sospesa nel futuro, resa particolarmente inquietante proprio dalla «normalità» dell'apparizione e dall'indeterminatezza, o forse meglio dalla sovradeterminazione, del suo significato: l'Uomo panciuto, ci avverte Marinetti nell'autocommento finale, «non è un simbolo» (non è, cioè, il Tempo, né la Vecchiaia né la Morte) «ma una sintesi alogica di molte sensazioni: paura della realtà futura, freddo e solitudine della notte, visione della vita 20 anni dopo, ecc.» (Marinetti, Settimelli e Corra, 1915: 33). Ma né queste né altre decine di proposte interpretative riuscirebbero a esaurire il senso di quel tocco di congedo sulle spalle dei giovani, più perentorio, nella sua levità, di qualsiasi anatema o profezia.

In conclusione di questo breve attraversamento del teatro fantastico futurista, ci sembra interessante ricordare che Anna Barsotti (Barsotti, 1990: 40-41, 140-141), riferendosi a un prodotto futurista leggermente più tardo, vale a dire il Manifesto del teatro visionico di Pino Masnata (1920), ne leggeva i motivi fondamentali, già presenti d'altronde in nuce nel teatro sintetico («la moltiplicazione della personalità, la soppressione della frontiera fra soggetto ed oggetto, e infine la trasformazione del tempo e dello spazio» (Barsotti, 1990: 40), alla luce di quelli che Tzvetan Todorov, nel suo libro fondativo sulla letteratura fantastica, ha classificato come «temi dell'io» (Todorov, 1977: 110-127). Sarebbe dunque proprio quest'apertura, inaugurata dal futurismo, alla «drammaturgia soggettiva» - che in Europa aveva già avuto un modello dirompente nell'opera di Strindberg e stava conoscendo importanti sviluppi sulla scena espressionista - a mettere una parte del teatro italiano su vie radicalmente alternative al realismo e a preparare terreni propizi al fantastico. Ne risentiranno ben presto tendenze drammaturgiche certamente innovative, ma meno scopertamente iconoclaste e «antiborghesi» del teatro futurista, a cominciare dal cosiddetto gruppo dei «grotteschi».

3.

La nozione di «teatro del grottesco», riferita a una tendenza drammaturgica sviluppatasi in Italia dalla metà degli anni Dieci del Novecento alla fine del decennio successivo, non è criticamente pacifica: nel senso che include tre autori (Luigi Chiarelli, Luigi Antonelli, Enrico Cavacchioli) tra loro notevolmente diversi negli obiettivi quanto nei mezzi per realizzarli, mentre una quarta figura (quella di Pier Maria Rosso di San Secondo) è addirittura da 
taluni considerata come la più rappresentativa della corrente, da altri del tutto irriducibile a essa, a causa della maggior altezza e complessità della sua opera, che la rende più difficilmente etichettabile. Esistono tuttavia certamente rilevanti tratti in comune nell'opera di questi drammaturghi: la loro capacità di «utilizzare in una testualità più complessa e in un circuito rappresentativo più regolare - almeno rispetto agli spettacoli futuristi - le intuizioni e le scoperte dell'avanguardia» (Barsotti, 1990: 136); il recupero di materiali tipici del teatro borghese (a partire dal triangolo moglie-marito-amante) per sottoporli a paradossali rovesciamenti ed esporli al ghigno tragicomico di una cinica, disincantata osservazione; il rapporto con il teatro di Pirandello, del quale in certi casi hanno il tempo di essere debitori, ma di cui più spesso anticipano motivi e strategie che poi il drammaturgo maggiore metabolizzerà con tutt'altra profondità e coerenza all'interno della propria opera. Infine, ed è ciò che qui più ci interessa, in tutti questi autori, pur in misure e modalità diverse «il tempo [...] si mostra fluidificato, liberalizzato, può accelerarsi o decelerarsi a piacere, in pratica risultare reversibile [...] il montaggio tende a farsi onirico [...] umiliando i principi razionali della non contraddizione, della causalità, della distinzione tra Soggetto ed Oggetto [...] il Personaggio risulta vanificato, $d i-$ sturbato, finisce per esplodere» (Puppa, 2003: 35): dominano, insomma, i «temi dell'io», e il teatro si apre all'irruzione del fantastico.

Il solo Chiarelli, tra i grotteschi, si mostra impermeabile a tale irruzione. In Cavacchioli, al di là di una serie di elementi che pertengono al grottesco senza poter essere ricondotti propriamente al fantastico (il personaggio del Meccanico, nella «visione in tre atti» Quella che t'assomiglia, del 1919, ha due ruote al posto degli occhi), l'accoglienza del fantastico si realizza soprattutto in una certa predilezione per la presenza di doppi perturbanti, siano essi di natura fantasmatica oppure macchinica: proprio nella pièce citata, all'ombra di Gabriele, che si aggira invisibile per la casa quando il personaggio è assente, fanno riscontro i fantocci automatizzati creati dal Meccanico. Senza mai sconfinare in modo esplicito nell'impossibile, il teatro di Rosso di San Secondo è tutto tramato di un'inquietante prossimità all'incubo. Già le sue giovanili Sintesi (1911), che nulla hanno a che spartire con quelle futuriste, si presentano come «proiezioni oniriche di un inconscio drammatizzato» (Cannavacciuolo, 2012: 43), in cui prevalgono le ambientazioni notturne e gli ingressi di certi personaggi hanno l'impatto di manifestazioni soprannaturali. Ed è a partire da questi brevi drammi che s'impone «quel costante riferimento all'«Assente», un personaggio che si costruisce attraverso le parole dei personaggi in scena cui vanno ricondotti i motivi dell'Attesa e dell'Apparizione inaspettata» (Can- 
navacciuolo, 2012: 46-47), che poi, amplificato fino all'ossessione, diventerà il tema centrale di una delle pièce più interessanti di Rosso, Lo spirito della morte (1931). ${ }^{8}$ E se la vicenda dello straordinario Ospite desiderato (1921) (ulteriore ripresa - forse inconsapevole - della situazione di base della Casa Usher di Poe genialmente rovesciata nei presupposti), «pur essendo essenzialmente umana, è nata da uno stato d'incubo» e «la realtà vi è, dunque, trasfigurata in sintesi allarmate e assorte come nei sogni tormentosi» (Rosso di San Secondo, 2008: 264), la protagonista del Segno verde (composto negli anni Trenta) si trova d'improvviso calata in una realtà enigmatica, dove ogni certezza si frantuma, le identità slittano e si sovrappongono, e personaggi reali si scoprono involontari protagonisti di una trama fantastica ideata da un romanziere che prodigiosamente li manovra come marionette viventi. Marionette, che passione! (1918) era d'altronde il titolo dell'opera più nota di Rosso, che, senza aperture al fantastico, già introduceva l'idea dei personaggi come creature eterodirette dalle proprie passioni e dai propri destini, in qualche modo simulacri di sé stessi, pietosi doppi di referenti umani che, in quanto padroni di sé e della propria vita, sono inesistenti. E come una «marionetta sonnambolica» (Bisicchia, 2009: 34) si muove sulla scena Carmelina, protagonista della Bella addormentata (1919), ibrido straordinario tra agghindata artificialità da bambola o da santa da sagra paesana e assonnata istintualità animalesca, «pupa grande» (Rosso di San Secondo, 2009: 250) che fa il suo ingresso in questa colorata fantasmagoria teatrale e da essa prende congedo come una sovrumana apparizione.

È Antonelli, tuttavia, il drammaturgo del «gruppo» che, con più chiara intenzionalità e continuità, ha fatto del fantastico la chiave di volta della propria opera (teatrale, ma anche narrativa), e secondo modalità che permetterebbero all'autore di "rivendicare [...] un proprio ruolo all'interno di quel grottesco novecentesco "neoromantico" e "modernista" che nel panorama europeo includerebbe, tra gli altri, surrealisti ed espressionisti: uno spazio, dunque, del tutto autonomo dalla tradizione italiana e che, proprio come la posizione di Pirandello, aspira a superare gli angusti confini della dimensione nazionale» (Giammarco, 1999: 181). Presente anche in una serie di opere minori, composte in buona parte tra gli anni Dieci e Venti, il fantastico teatrale di Antonelli raggiunge i suoi risultati più compiuti e originali in una trilogia di «avventure fantastiche» costituita da L'uomo che incontrò se stesso

8 «[...] insistito risulta il motivo della camera in attesa, ossia dello spazio magico-allucinatorio per la rievocazione, meglio per la resurrezione dell'assente. Il vivo diventa, in tal caso, un maniacale officiante d'un rito animistico, d'una macabra messinscena in cui gli altri personaggi intervengono quali agenti subalterni per l'epifania dello spettro» (Puppa, 1985: 37). 
(1918), La bottega dei sogni (1927) e La rosa dei venti (1928), tematicamente e strutturalmente simili, e in una commedia del 1924, La casa a tre piani. Il motivo unificante delle «avventure» (l'approdo del protagonista in un luogo appartato e sconosciuto al mondo reale, e governato da un personaggio - mago, scienziato o dio - dotato di poteri soprannaturali), sembrerebbe inclinare il teatro antonelliano verso una dimensione magico-fiabesca (inclinazione ancor più chiaramente presente, già dal titolo, in altre opere, come La fiaba dei tre maghi del 1919, e I diavoli nella foresta, del 1920) sottraendolo perciò alla modalità propriamente fantastica. In realtà l'impostazione di genere féerique, indubitabilmente presente sia nelle «avventure fantastiche» che in altre pièce, è turbata dall'interno da segnali di incredulità, esitazione, inquietudine, paura che, almeno per parte dell'azione, escludono quella tranquilla accettazione del prodigio che ascriverebbe senza mezzi termini questo teatro al meraviglioso. ${ }^{9}$

Per esemplificare sulla prima, che rimane forse anche la più riuscita, opera della trilogia, L'uomo che incontrò se stesso, già una singolare espressione presente nella didascalia iniziale annuncia l'intenzionale iniezione di un perturbamento fantastico all'interno della dimensione fiabesca: l'ambientazione, si dice, sarà un «paesaggio irreale [...] caratterizzato da grandi cipressi color viola che danno alla scena un aspetto violento, una intonazione e un incubo di fiaba» (Antonelli, 1994: 9; corsivo nostro). A parte il fatto rilevante che, qui come in altre pièce di Antonelli, l'alterità rispetto alla realtà quotidiana è affidata in prima istanza a indicazioni scenografiche e cromatiche, ${ }^{10}$ la locuzione che abbiamo evidenziato indica come la fiaba sia in realtà il prodotto di un incubo e che di conseguenza una sensazione di disagio e inquietudine la abita in profondità. L'atmosfera misteriosa e come sospesa dell'isola in cui il protagonista Luciano si ritrova senza sapere come vi è giunto è d'altronde alquanto unheimlich, e le prime indirette notizie che egli riceve sul proprietario del luogo, il dottor Climt, sono tutt'altro che rassicuranti: il primo essere vivente che incontra, la giovane Rosetta, lo avverte che

9 È d'altra parte una caratteristica propriamente «novecentesca» di queste opere la compresenza e la commistione del fiabesco, dell'umoristico e del fantastico: che a volte si alternano o si equilibrano felicemente, mentre in altri casi possono effettivamente produrre l'impressione di un'irrisolta armonizzazione delle singole tonalità.

10 La prima didascalia della Bottega dei sogni dà indicazioni molto simili: «si profilano contro il cielo di porpora [...] le alte cime dei cipressi, simili a enormi ceri violacei [...] Qualcosa d'irreale, quasi di argenteo, diffonde sullo scenario l'incubo e la leggerezza d'una favola» (Antonelli, 1994: 67). Un'atmosfera visiva oniricamente inquietante è prevista anche dall'inizio della didascalia d'apertura della Rosa dei venti: «Grande salone architettonico a piani sintetici e volute ampie e leggere su cui pare aleggi l'incubo immateriale del Tempo» (Antonelli, 1994: 121). 
Climt è pericoloso e lo invita a tenersene alla larga, descrivendo così i suoi poteri: «si diverte con gli uomini come un fanciullo coi burattini. È il suo segreto. È un uomo terribile, vi dico! Egli può tutto» (Antonelli, 1994: 12). Quando poi la donna gli dice che conosce Climt da più di un secolo, Luciano reagisce in modo sensatamente razionale, e insomma non «fiabesco», convincendosi che Rosetta sia pazza. La comparsa di Climt, elegante ma in modo troppo impeccabile, gentile ma con un eccesso di freddezza, lo sguardo penetrante nel volto pallidissimo, ${ }^{11}$ serve più a rafforzare che a dissipare i timori e le incertezze di Luciano: che si accresceranno ulteriormente quando, accortosi che il suo orologio si è stranamente fermato, apprenderà che sull'isola non esistono orologi. L'inizio, a ben guardare, potrebbe perfettamente attagliarsi a una storia fantastica d'intonazione horror, il cui protagonista capita in un luogo ai margini del mondo, dove aleggia la presenza di un mistero di cui non gli vengono offerti che alcuni perturbanti segnali, ai quali cerca inizialmente di reagire con lo scetticismo della ragione. Ben presto però le cose evolveranno effettivamente in un'altra direzione, dal momento in cui si scoprirà che Climt è effettivamente uno scienziato-mago che possiede il potere di fermare o invertire il tempo, e Luciano, vinte le ultime resistenze razionali, gli crederà fino al punto di accettare l'incontro con il sé stesso di vent'anni prima per tentare di correggerne l'infausto destino sentimentale. Per dirla con le categorie di Todorov, si passa da una prima fase fantastica, caratterizzata dall'esitazione interpretativa nei confronti degli eventi, all'entrata nella dimensione del meraviglioso, dove l'esistenza di fatti estranei alle leggi di natura non costituisce più alcuno scandalo agli occhi dei personaggi. Nel caso specifico di questo lavoro, poi, il pieno ingresso nel fiabesco coincide con l'accentuazione della componente umoristica (qui in gran parte dedicata alla parodia del triangolo amoroso borghese), che nella prima breve fase fantastica, anche per non guastarne la suggestione inquietante, si era limitata a una sottile patina ironica. C'è a nostro avviso, in questo gioco di toni e generi, una notevole tensione sperimentale, che volutamente non persegue l'armonizzazione completa dei diversi elementi; anche se è comprensibile che il risultato possa sembrare in definitiva fallimentare,

11 Sul carattere ambiguo, ma fondamentalmente demoniaco, di Climt, si sofferma M. Giammarco: «lo statuto del dottor Climt conserva una certa apparenza da scienziato-stregone di tipo faustiano, compromesso con forze diaboliche, forze del male che promettono all'uomo, con l'elisir magico, l'immortalità e l'eterna giovinezza, l'esercizio di poteri sovrumani in contrasto con le leggi divine. L'ambiguità della sua natura, che è alla base dell'esitazione fantastica che circola nel testo (condita sempre, tuttavia, dell'umorismo antonelliano), affiora già dall'aspetto fisiognomico» (Giammarco, 1999: 193-194). 
se invece lo si attribuisce a un'«incapacità di Antonelli di costruire, e poi reggerla fino in fondo, un'atmosfera davvero surreale e onirica» (Ariani-Taffon, 2001: 43).

Vi è comunque almeno un'opera, nel corpus drammaturgico antonelliano, nella quale il fantastico si presenta senza compromissioni col fiabesco, introducendo in un tessuto di normalità quotidiana, e non in una dimensione fin da subito caratterizzata da elementi di onirismo o magismo, la componente fantastico-perturbante. Si tratta della già citata Casa a tre piani, che già nell'ambiente descritto dalla didascalia d'apertura rivela la propria distanza dalle «avventure»: «Una vasta camera da pranzo, nel primo piano della casa. Tavola imbandita. Si è alla fine del pranzo. Chi fuma, chi prende il caffè. [...] Da una finestra, che è aperta sulla parete sinistra, si respira l'aria di una serata estiva» (Antonelli, 1994: 195). Nulla di anormale, dunque, almeno all'apparenza, in questo interno dove gli inquilini stanno festeggiando il centenario della costruzione dell'edificio. Ma già il discorso celebrativo dell'Avvocato introduce un elemento perturbante nel suggerire un'implicazione particolare tra la «vita» della casa e quella dei suoi abitanti: «Tutti noi abbiamo amata e amiamo la sua intimità, le cicatrici del tempo, le macchie dell'umido, le ferite della folgore che la colpì, il tremito delle sue persiane, le tappezzerie delle sue pareti, e tutti i pallidi volti qua e là impressi negli specchi che non dimenticano» (Antonelli, 1994: 196). Se non si tratta di una maison propriamente hantée, è tuttavia più che lecito il suo accostamento a certe case del «Teatro da camera» di August Strindberg (1907), «dove la simmetria degli spazi e la loro simultanea esistenza alludono alla geometria dei personaggi» (Sammartano, 1994: XXXII), e la reciproca implicazione simbolica tra gli edifici e i personaggi sfiora l'identificazione magica. In Antonelli, il personaggio delegato a sintonizzarsi con l'anima segreta della casa è una ragazza, denominata «la Spiritata», che vive solitaria al terzo piano e che pare dotata del dono della profezia. «La casa è pura soltanto lassù... dove la terrazza respira il suo cielo!», spiega la ragazza agli altri inquilini, in parte scettici e in parte turbati. «E il cielo benedice la casa quando quelli che la abitano possano guardarlo con limpidi occhi! [...] Se una creatura soffre, la casa è in peccato...» (Antonelli, 1994: 201). Toni e contenuti si fanno più perturbanti durante la trance profetica della spiritata: «Sento che si lagnano. È la casa che patisce... (con terrore) e si lamenta anche, pur conservando il suo volto impassibile. [...] tutto torna a battere, a battere... È come un polso! La casa ha il suo polso implacabile! E quel che si rannicchia negli angoli è sempre il tradimento che cova, e non bastano i balconi aperti per tirarlo fuori...» (Antonelli, 1994: 203). 
In relazione agli effettivi poteri divinatori della Spiritata, gli atteggiamenti dei condòmini sono differenziati, ma, a parte gli scongiuri superstiziosi della Beghina, risultano in maggioranza improntati allo scetticismo o al massimo a un'apertura possibilista alla comprensione da parte di una ragione «allargata» rispetto ai confini angusti della scienza attuale: posizione, quest'ultima, sostenuta dal Dottore, che ammette l'esistenza di «tipi privilegiati, o morbosi», che "sono le creature della nostra congiunzione, se si può dire, col mondo imperscrutabile, ossia con quello che non cade sotto il controllo dei nostri sensi», ma premurandosi di ricondurlo al caso clinico («un tipo interessantissimo di isterica epilettoide») o ai meccanismi del subconscio («la visione telepatica degli avvenimenti» spiegata come «un fenomeno di subcoscienza» (Antonelli, 1994: 199). Fatto sta che, una volta che la Spiritata ha profetizzato l'imminente morte di uno degli inquilini, senza essere in grado di precisarne l'identità, l'atteggiamento superstizioso, dichiarato o camuffato, dilaga tra i personaggi, ognuno timoroso (o speranzoso, nel caso dell'Uomo che avrebbe già dovuto morire, malato e impoverito) di essere la vittima designata. Questa è invece proprio la Spiritata, la quale, presa coscienza del proprio imminente destino, nel suo luminoso appartamento, più vicino al cielo che al tragicomico teatrino di egoismi e meschinità che si agita ai piani inferiori, ha indossato una veste elegante, come chi si prepara a ricevere ospiti; e si pone in attesa, in compagnia della Nonna, sordomuta e immobile come un «vecchio idolo» (Antonelli, 1994: 225), di Colei che «tante volte, quando non aveva fretta, sì è seduta là, e poi se n'è andata» (Antonelli, 1994: 233). Si riconosce ancora una volta, nella scena finale di progressivo appressamento, piano per piano, pianerottolo per pianerottolo, della Morte con il suo «leggero, il suo formidabile passo» (Antonelli, 1994: 234), il modello dell'Intrusa di Maeterlinck. E, come nel dramma dello scrittore belga, tutto avviene senza che nulla di impossibile si offra alla percezione dei sensi corporei, sicché la pièce di Antonelli può chiudersi in questo caso, con grande sobrietà di mezzi, nel segno del puro fantastico: «Improvvisamente si aprono i due battenti della porta, come se una folata di vento li spingesse. Si vede il pianerottolo buio» (Antonelli, 1994: 234). Il vento? Suggestione? Una pura coincidenza? Vero è comunque che la Spiritata «ha appoggiato la nuca sulla spalliera della poltrona e non si muove più» (Antonelli, 1994: 234.).

4.

L'unico (con l'eccezione, parziale e del tutto particolare, di Pirandello) tra i drammaturghi italiani del primo Novecento che abbia riservato alla 
componente fantastica un ruolo rilevante all'interno del proprio teatro e che sia stato diffusamente riconosciuto, in antologie e studi critici, come un esponente del modo letterario in questione è Massimo Bontempelli: anche se questo avviene più per la sua narrativa che per l'opera drammatica, in ragione del fatto che, in generale, gli strumenti critici adatti ad affrontare il fantastico in ambito teatrale rimangono a tutt'oggi meno affinati e precisi di quelli da tempo applicati alle forme narrative. Rimane inoltre la difficoltà, per scrittori come Bontempelli (ma anche Pirandello) che hanno infuso la loro predilezione per il fantastico nella produzione teatrale quanto in quella narrativa, di saldare i due momenti in modo da salvarne tanto la continuità quanto le specificità legate alla differenza dei codici espressivi. Altra particolarità di Bontempelli, rispetto al resto degli autori da noi presi in considerazione, è quella di essere l'unico ad avere fornito un sostrato teorico alla propria declinazione del fantastico, attraverso la nozione di «realismo magico», frutto di una ricerca del versante «miracoloso» del reale che nulla deve affidare all'arbitrarietà: «Precisione realistica di contorni, solidità di materia ben poggiata al suolo; e intorno come un'atmosfera di magia che faccia sentire, traverso un'inquietudine intensa, quasi un'altra dimensione in cui la vita nostra si proietta» (Bontempelli, 1974: 351). Il «progetto» fantastico di Bontempelli, in completa autonomia rispetto ai residui romantici ma anche alle atmosfere simboliste, non si propone di perseguire effetti decisamente perturbanti né tantomeno terrorizzanti, ma di realizzarsi piuttosto in una sottile (e però intensa) inquietudine, derivante da un'osservazione lucida e oggettiva, quasi imperturbabile, degli sconfinamenti magici della realtà. Alla clarté e al nitore della scrittura fantastica bontempelliana, e a quella sua "precisione realistica di contorni», non rimarrà insensibile Italo Calvino, che nella terza delle sue Lezioni americane (dedicata, non a caso, all'«Esattezza») includerà Bontempelli, unico italiano, in un consesso di tutto rispetto (comprendente tra gli altri Paul Valéry, Fernando Pessoa e Jorge Luis Borges), accomunato dalla «rivalutazione dei procedimenti logico-geometrici-metafisici» (Calvino, 1988: 69) e dall'impiego di una scrittura "cristallina»: «Il cristallo», dichiara Calvino, «con la sua esatta sfaccettatura e la sua capacità di rifrangere la luce, è il modello di perfezione che ho sempre tenuto come un emblema» (Calvino, 1988: 69); emblema almeno in un'occasione esplicitamente condiviso da Bontempelli, per il quale il grande scrittore (Pirandello, nella fattispecie) sa leggere nel groviglio del mondo e schiarirlo «fin che esso tutto appare fuori dalle ultime nebbie come una costruita fantasia di cristalli di sale» (Bontempelli, 1978: 821). 
Se è vero che il progetto di un realismo magico attinto attraverso la rarefatta precisione della scrittura ha più agio di realizzarsi nella narrativa, soprattutto nelle due «favole metafisiche» La scacchiera davanti allo specchio (1921) ed Eva ultima (1922), grazie anche all'evocazione descrittiva di un paesaggio "ridotto ad un minimalismo di contorni e di linee», e dunque a «"metafisica della luce", stasi, nirvanica infinità» (Bellotto, 2003: 151), il teatro non vi rimane affatto estraneo. Ma esso è forse ancor meglio leggibile alla luce di un'altra nozione fondante per la poetica bontempelliana, che è quella di «candore», inteso come la prerogativa, di cui alcuni (pochi) individui sono dotati, «di saper maravigliarsi, di sentire che l'universo, e tutta la vita, sono un continuo inesauribile miracolo» (Bontempelli, 1974: 353). Incauta per vocazione e indisponibile ai compromessi, «l'anima candida, che è una forza elementare, va facilmente al fondo delle cose [...] È piena di senso del mistero [...] e se ne fa custode per gli altri uomini» (Bontempelli, 1978: 812-813). Candore è dunque anche la facoltà su cui si basa l'attitudine al fantastico, la capacità di scorgere, oltre le convenzioni, le sovrastrutture, le ipocrisie, il fondo magico (che può però essere anche tragico) della realtà.

Candidi sono alcuni grandi scrittori: da Lucrezio a Pirandello, passando per san Francesco e Leopardi. Ma tra i personaggi bontempelliani, quelli teatrali in particolare, il candore è appannaggio quasi esclusivo delle donne. La drammaturgia fantastica di Bontempelli, che, se si eccettua la favola allegorica di Nembo (1935), si concentra in quattro opere composte tra gli anni Dieci e i Venti (La guardia alla luna, del 1916, Siepe a nordovest, del 1919, Nostra Dea, del 1925, e Minnie la candida, del 1927), presenta infatti una serie esemplare di anime candide. ${ }^{12}$ Le protagoniste della prima e dell'ultima pièce, in particolare, sono emblemi di come il candore possa essere anche feroce, indomabile, caparbio e irragionevole come la follia. Maria, la protagonista della Guardia alla luna, e Minnie sono entrambe divorate da un'ossessione in apparenza del tutto irragionevole: la prima, in seguito alla morte della sua bimba, si convince che la luna favorisca i concepimenti solo per poter poi rapire i bambini, di cui la sua luce si nutre, e si pone come missione di impedire che $\mathrm{i}$ raggi del satellite raggiungano la terra; l'altra, in seguito a una frase scherzosa sull'esistenza di automi fuggiti da un laboratorio, che si credono veri uomini e da essi sono indistinguibili, cade preda dell'idea fissa che il mondo sia pieno di questi spaventosi simulacri, fino a barricarsi in casa per sottrarsi al loro

12 La presenza e il ruolo delle «candide» nel teatro di Bontempelli sono distesamente analizzati in Manfrini, 2003-2004. 
assedio, e decidere infine di suicidarsi quando la sua immagine allo specchio la convince di essere lei stessa una «replicante» ${ }^{13}$ in cui sia stata inserita la memoria di una vita vera. Ma la follia di entrambe è candore, cioè visione profonda di verità nascoste e terribili: in un caso il volto doppio e spaventoso della femminilità materna, potenziale divoratrice delle proprie creature, nell'altro il claustrofobico e assediante sentimento di una crescente disumanizzazione e perdita di autenticità.

In Minnie la candida, come si è visto, l'ossessione della protagonista ha per oggetto un tipico motivo del fantastico perturbante, la presenza del simulacro e la difficoltà di distinguere tra gli essere viventi e i loro doppi artificiali: Bontempelli, pur non intendendo evidentemente convincere il lettore e lo spettatore della letterale verità della convinzione di Minnie, coglie comunque le potenzialità unheimlich derivanti dalla situazione, accentuando nelle persone osservate con sospetto da Minnie quei tratti di meccanicità o di eccessiva perfezione che la donna tende a leggere come segnali di riconoscimento dei simulacri. Se poi a questo si aggiungono coincidenze che non sembrano casuali, la vera e propria esitazione fantastica può far capolino per un istante: come quando, poco dopo che Minnie ha appreso la falsa notizia che gli automi scappati dal laboratorio sono dodici, metà maschi e metà femmine, «entrano da sinistra SEI UOMINI $e$ SEI DONNE in tenuta di globe-trotter e quasi a passo di marcia silenziosamente traversano la scena e scompaiono a destra» (Bontempelli, 1947, I: 234).

Con tutt'altri esiti - di leggera, umoristica surrealtà - l'idea di simulacri artificiali incoscienti di esserlo aveva già trovato attuazione in Siepe a nordovest: si trattava, in quel caso, di marionette, pienamente e tronfiamente convinte di essere uomini. Ma la particolarità più originale di questa pièce consiste nel fatto che, condividendo lo stesso spazio, la troupe di marionette e un gruppo di persone in carne e ossa agiscono a contatto di gomito senza potersi vedere, ma percependo e potendo anche utilizzare in comune gli oggetti materiali che si trovano in scena, con la conseguenza di buffi (per il pubblico) e inquietanti (per i personaggi, umani e artificiali) incidenti che, per le marionette in particolare, hanno l'aria di prodigi: per loro, assai più che per lo spettatore, gli avvenimenti recano la marca del fantastico. La figura di una candida appare qui

13 Non è questo, naturalmente, il termine impiegato da Bontempelli, essendo stato coniato dai creatori del film Blade Runner (1982), di Ridley Scott: ma è evidente come il delirio di Minnie precorra temi diffusi dalla posteriore fantascienza, rivelando in particolare singolari somiglianze con alcune opere di Philip K. Dick, tra cui il romanzo Do Androids Dream of Electric Sheep? (1968), dal quale Blade Runner deriva. L'incubo di una progressiva, inarrestabile invasione di una genia simil-umana che si sostituisce alla vera umanità è il tema anche di un precedente capolavoro del cinema fantascientifico, Invasion of the Body Snatchers (1956), di Don Siegel, a sua volta derivato dal romanzo di Jack Finney The Body Snatchers (1955). 
solo nel finale nelle sembianze di una Zingara, unico personaggio in grado di scorgere tanto gli uomini che le marionette, perché provvisto di uno sguardo che non si limita alla propria verità «regionale», ma sa allargarsi alla percezione simultanea di due diversi ma contigui livelli di realtà. E ancora marionetta, ma di tutt'altra specie, è Dea, protagonista della commedia del '25: in una variante ironica e satiricamente aggiornata del motivo fantastico del vestito incantato o stregato, che «possiede» chi lo indossa, la donna muta di personalità a ogni cambio d'abito. Ora dolce e protettiva, ora aggressiva e passionale (Manfrini, 2003-2004: 51), Dea attraversa anche fasi più inquietanti: una spossatezza che l'avvicina all'insensibilità e alla morte, quando è coperta da uno scialle color cenere; ma anche la natura spaventosa della serpe o del drago, con lo sguardo saettante e la voce piena di sibili, se indossa una veste «di squame verdi luccicanti aderentissime». Ma il mutamento più perturbante è forse il finale ritorno, una volta svestita, alla condizione di manichino dai movimenti meccanici, con la voce apatica che sillaba in modo automatico qualche ultima parola prima di riconsegnarsi, novecentista Olimpia hoffmanniana, alla definitiva inerzia di una bambola uscita dal gioco.

\section{BIBLIOGRAFIA}

ANTONELLI, Luigi (1994): L'uomo che incontrò se stesso ed altri drammi rappresentati 19181933, a cura di Giancarlo Sammartano, Bulzoni, Roma.

ARIANI, Marco - TAFFON, Giorgio (2001): Scritture per la scena. La letteratura drammatica nel Novecento italiano, Carocci, Roma.

Artioli, Umberto (1975): La scena e la dynamis, Pàtron, Bologna.

BArsotti, Anna (1990): Futurismo e avanguardie nel teatro italiano fra le due guerre, Bulzoni, Roma.

Bellotto, Silvia (2003): Metamorfosi del fantastico. Immaginazione e linguaggio nel racconto surreale italiano del Novecento, Pendragon, Bologna.

Bertolotti, Costanza (2013): «Simpatie e affinità misteriose. Futurismo, anarchismo, occultismo: l'esperienza della folla», in Vinzia Fiorino, Gian Luca Fruci, Alessio Petrizzo (edd.), Il lungo Ottocento e le sue immagini. Politica, media, spettacolo, ETS, Pisa, pp. 115-130.

Bisicchia, Andrea (2009): «I testi», in Pier Maria Rosso di San Secondo, Tutto il teatro, vol. II. Rosso di San Secondo e il teatro del colore, a cura di Andrea Bisicchia, Salvatore Sciascia, Caltanissetta-Roma.

Bontempelli, Massimo (1947): Teatro, 2 voll., Mondadori, Milano.

(1974): L'avventura novecentista, Vallecchi, Firenze.

(1978): Pirandello o del candore, in Id., Opere scelte, a cura di Luigi Baldacci, Mondadori, Milano, pp. 809-828. 
ButTi, Enrico Annibale (1910): Il castello del sogno, Treves, Milano.

Calvino, Italo (1988): Lezioni americane, Garzanti, Milano.

Cannavacciuolo, Laura (2012): La fabbrica del grottesco. Il teatro di Chiarelli, Rosso di San Secondo, Cavacchioli e Antonelli, Tullio Pironti, Napoli.

Cavacchioli, Enrico (1990): L'uccello del paradiso e altri drammi rappresentati 1919/1929, a cura di Giancarlo Sammartano, Bulzoni, Roma.

CiglianA, Simona (2006): «Letteratura, spiritualismo, occultismo tra le due guerre», in Gianfranco de Turris (ed.), Esoterismo e Fascismo. Storia, interpretazioni, documenti, Edizioni Mediterranee, Roma, pp. 197-221.

De Antonellis, Gianandrea (2012): Enrico Annibale Butti. L'Ibsen italiano, Esi, Napoli.

Giammarco, Marilena (1999): «Un mago sulla scena italiana del Novecento: il dottor Climt di Luigi Antonelli», in Rosalba Gasparro e Roberta Mullini (edd.), Il meraviglioso teatrale tra fiaba e magia, Edizioni Tracce, Pescara, pp. 179-209.

MANFrini, Silvia (2003-2004): Tra follia e saggezza: le candide e le innocenti nel teatro di Bontempelli, tesi di laurea quadriennale, Università di Verona, Corso di laurea in Lettere.

Marinetti, Filippo Tommaso, Settimelli, Emilio e Corra, Bruno (1915): Teatro futurista sintetico, vol. I, Istituto Editoriale Italiano, Milano.

(1969): La grande Milano tradizionale e futurista. Una sensibilità italiana nata in Egitto, Mondadori, Milano.

Melani, Costanza (2006): Effetto Poe. Influssi dello scrittore americano sulla letteratura italiana, Firenze University Press, Firenze.

Pedullà, Gabriele (2010): Pirandello, o la tentazione del fantastico, in Luigi Pirandello, Racconti fantastici, a cura di Gabriele Pedullà, Einaudi, Torino, pp. V-XXXII.

Poe, Edgar Allan (1982): The Complete Tales and Poems of Edgar Allan Poe, Penguin Books, London.

Puppa, Paolo (1985): Rosso di San Secondo: per un nuovo Teatro Stoico, in Eugenia Ocello (ed.), Il teatro di Rosso di San Secondo, Franco Cesati, Firenze. (2003): Il teatro dei testi. La drammaturgia italiana nel Novecento, UTET, Torino.

Rosso di SAN SECondo, Pier Maria (2008): Tutto il teatro, vol. I. La dimensione europea, a cura di Andrea Bisicchia, Salvatore Sciascia, Caltanissetta-Roma.

(2009): Tutto il teatro, vol. II. Rosso di San Secondo e il teatro del colore, a cura di Andrea Bisicchia, Salvatore Sciascia, Caltanissetta-Roma.

SAMmARTANo, Giancarlo (1994): «Introduzione», in Luigi Antonelli, L'uomo che incontrò se stesso ed altri drammi rappresentati 1918-1933, a cura di Giancarlo Sammartano, Bulzoni, Roma.

Todorov, Tzvetan (1977): La letteratura fantastica, Garzanti, Milano (edizione originale: Introduction à la littérature fantastique, Editions du Seuil, Paris, 1970).

Verdone, Mario (1969): Teatro del tempo futurista, Lerici, Roma. 